\title{
Trigeminal Neuralgia: The pain is a given, the cause is not
}

\author{
Sophia Vassilopoulou ${ }^{1 *}$, Argyro Tountopoulou ${ }^{1}$, Eleni Korompoki ${ }^{2}$, Loukia Poulou ${ }^{3}$ and Christos Gkogkas ${ }^{4}$ \\ ${ }^{1} 1$ st Department of Neurology, Eginition Hospital, National and Kapodistrian University of Athens, Greece \\ ${ }^{2}$ Department of Clinical Therapeutics, National and Kapodistrian University of Athens, Greece \\ ${ }^{3}$ Radiology Department, Iaso Hospital, Athens Greece \\ ${ }^{4}$ Interventional Neuroradiology Department, Metropolitan General Hospital, Athens Greece
}

\begin{abstract}
Trigeminal neuralgia is a severe facial pain. Dural Arteriovenous Fistula is a very rare cause of this neuralgia. We present a 68 year old male with a severe left

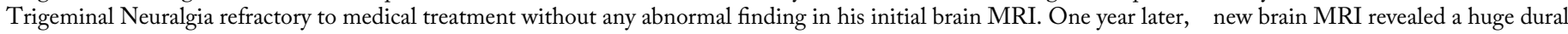
Arteriovenous Fistula (AVF) as the underlying cause of this painful prosopalgia. Successful transarterial dAVF embolization improved patient's symptoms and subsequently his quality of life
\end{abstract}

\begin{abstract}
Abbreviations: dAVF: Dural Arteriovenous fistula; AVM: Arteriovenous Malformation; MRI: Magnetic Resonance Images SCA: Superior Cerebellar Artery; AICA: Anterior Inferior Cerebellar Artery; DSA: Digital Subtraction Angiography
\end{abstract}

\section{Introduction}

Trigeminal neuralgia (TN) is a severe, paroxysmal, facial pain with an incidence of 4-7/100.000 [1]. Its main cause is a vascular compression at the entry zone of the Trigeminal nerve by an aberrant loop of an artery or a vein in the posterior fossa [2].

The secondary causes are rare and include multiple sclerosis, tumors and Arteriovenous Malformation (AVM) [3].

We present a patient who suffered over a year from TN due to a gradually growing dural Arteriovenous Fistula (dAVF) [4] which hadn't been detected in the first brain MRI and soon became huge causing symptoms and signs unbearable to the patient.

\section{Case study}

A 68- year -old male presented with a severe, rapid, electric shock pain in the second branch of his left Trigeminal nerve. His brain MRI didn't reveal any abnormal findings. He was initially treated with carbamazepine up to $800 \mathrm{mg} /$ day. A week later, though the pain was relieved, the patient reported dizziness and gait disturbances as an adverse effect from the carbamazepine and the treatment switched to gabapentin and amitryptilline up to the maximum dose. The symptoms were well controlled on this treatment, although the patient referred some, short duration, episodes of remission. One year after, the pain worsened and didn't respond to the maximum dose of any pain relief medication (antiepileptic, NSAIDs, opioids, combination). The patient complained of dizziness, ataxia, confusion and, sometimes, speech difficulties, which were all attributed to medication adverse effect. Therefore, the neurosurgical intervention was regarded as a possible therapeutic option. A new brain MRI then was performed and surprisingly a huge dAVF in the left transverse sinus, associated with diffuse venous reflux into subarachnoid veins resulting in brainstem compression at the exit of the left trigeminal nerve, was detected (Figures 1-6). The patient was successfully treated with transarterial embolization with liquid embolic agent (Onyx) and the dAVF was completely occluded (Figures 7-9). Our patient slowly improved and 6 months later was free of symptoms and free of any medication.

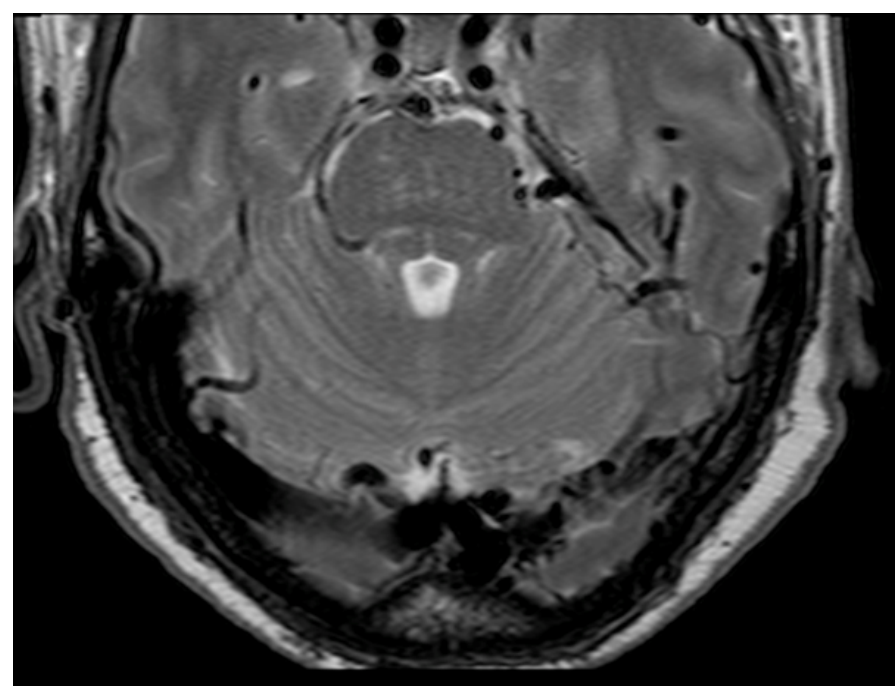

Figure 1. MRI- Axial T2 weighted image at the level of the pons demonstrates enlarged veins (arrow) at the root entry zone of left trigeminal nerve

*Correspondence to: Vassilopoulou S, MD, PhD, Assistant Professor of Neurology, 1st Department of Neurology, Eginition Hospital, National and Kapodistrian University of Athens, Greece, Tel: +30210-7289290; Fax: +30210-7216474; E-mail: svassilopoulou@gmail.com

Key words: trigeminal neuralgia, dural arteriovenous fistula, venous reflux, brainstem compression

Received: April 01, 2020; Accepted: April 14, 2020; Published: April 17, 2020 


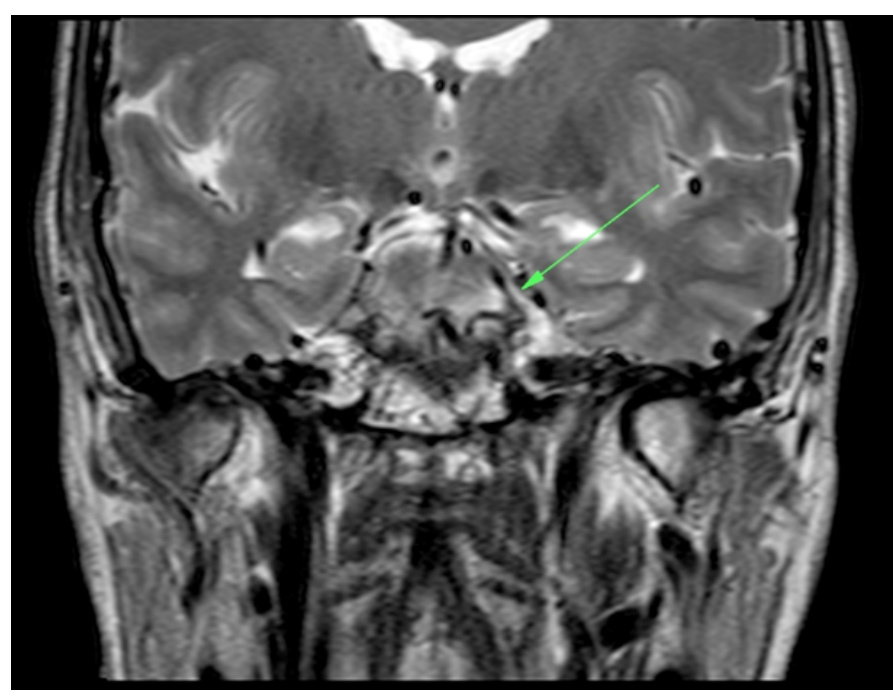

Figure 2. MRI- Coronal T2 weighted image at the level of the pons demonstrates enlarged veins (arrow) at the root entry zone of left trigeminal nerve

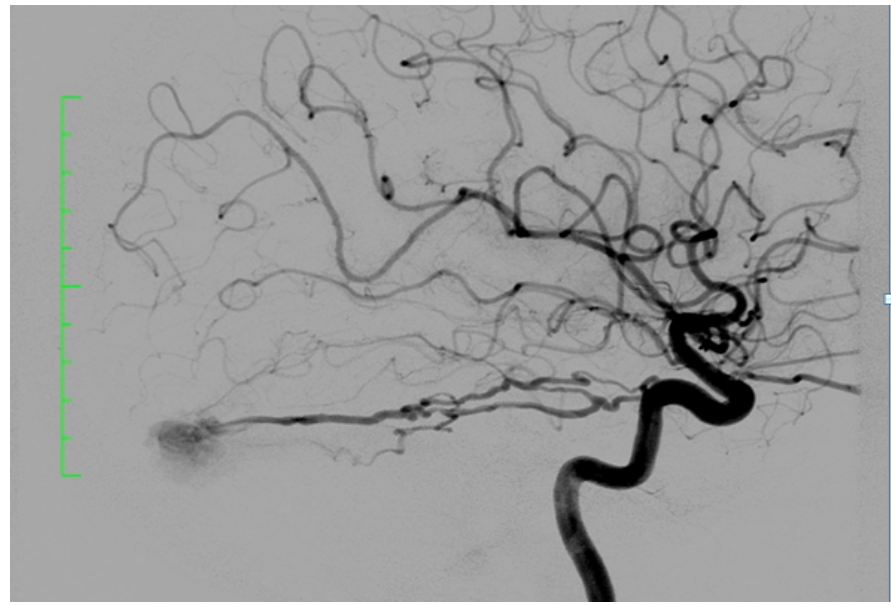

Figure 3. Lateral projection LICA pre- embolization DSA (arterial phase) demonstrates early filling of the left transverse sinus through branches of the meningohypophyseal trunk

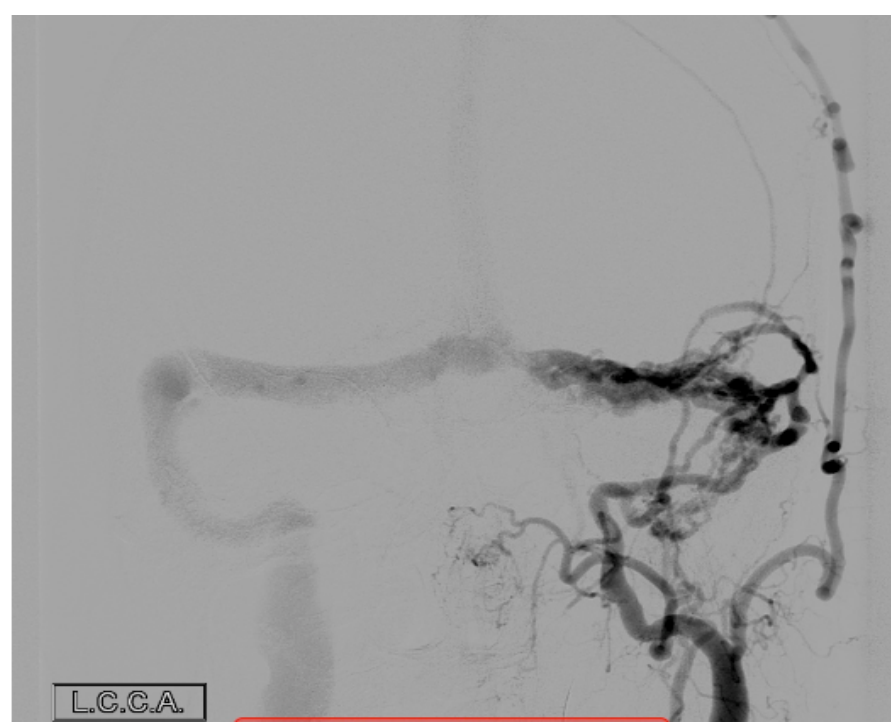

Figure 4. Anteroposterior projection left external carotid artery pre- embolization DSA demonstrates early filling of the transverse sinus through occipital artery and middle meningeal artery branches

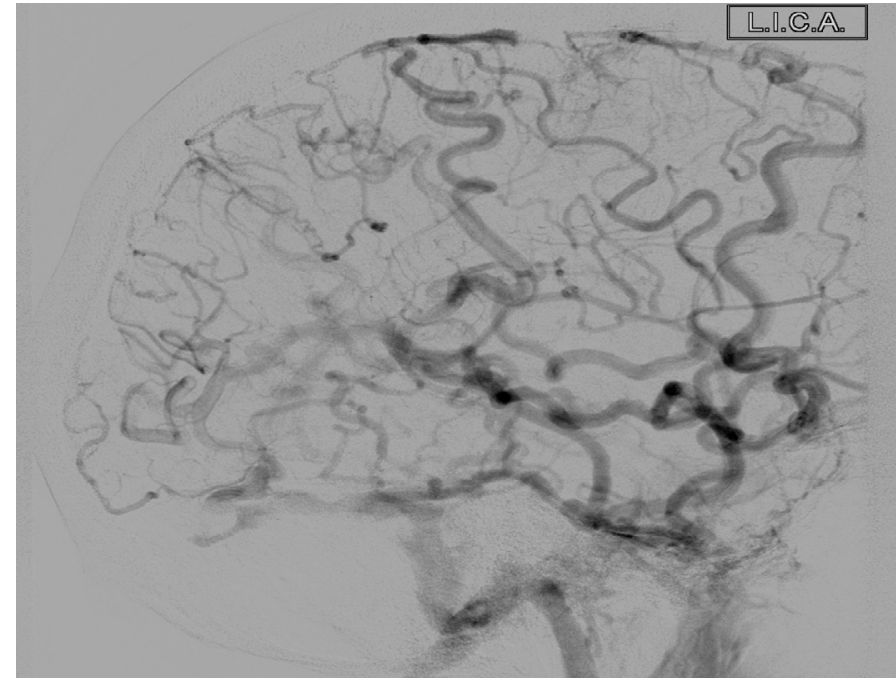

Figure 5. Lateral projection LICA pre- embolization angiogram(venous phase) demonstrates significant venous congestion

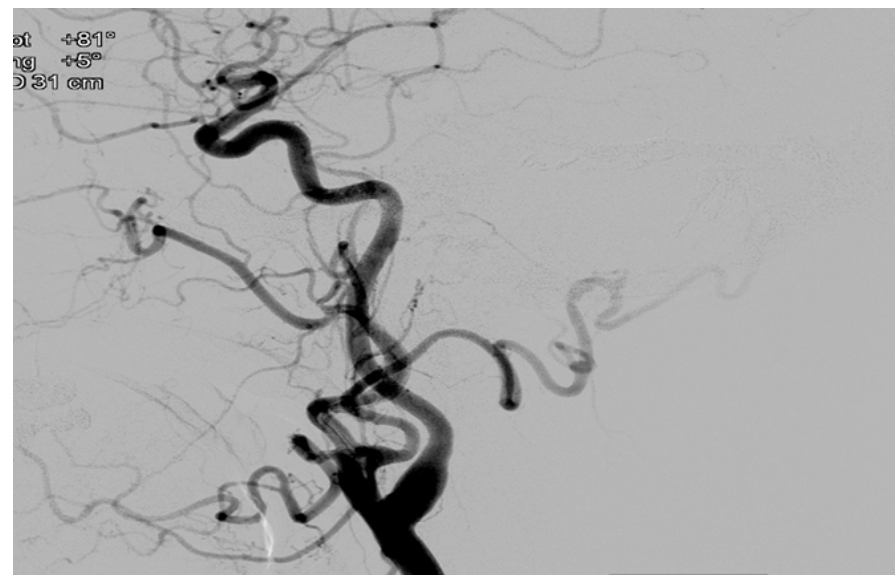

Figure 6. Anteroposterior LVA pre embolization DSA demonstrates early venous filling of the torcular and right transverse sinus associated with reflux into the superior sagittal sinus. Bihemispheric pial venous congestion is also demonstrated

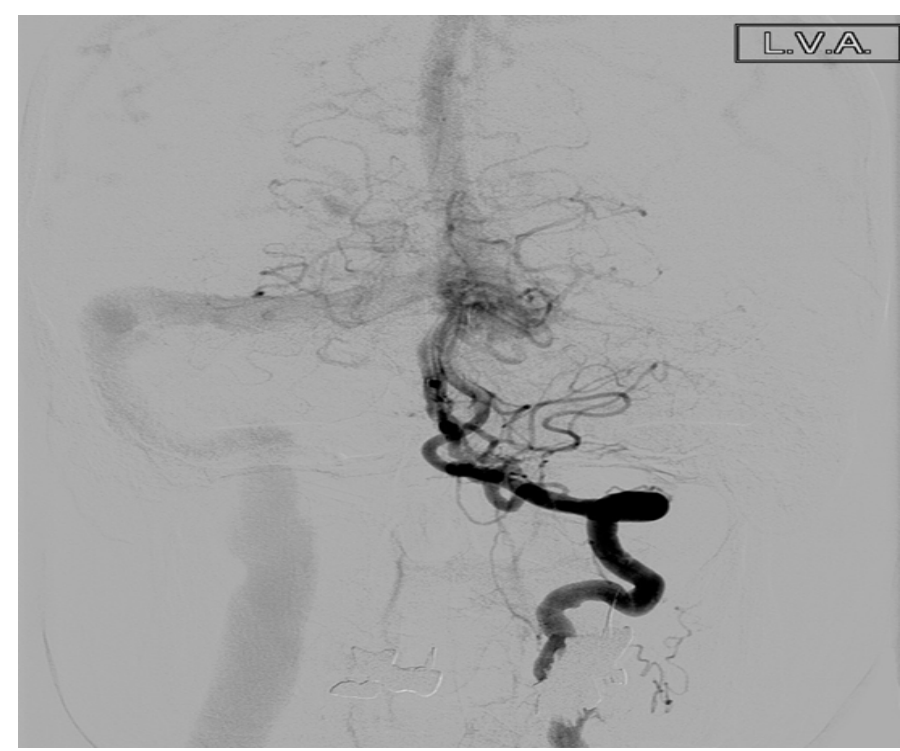

Figure 7. Lateral projection LCCA post- embolization DSA demonstrates complete obliteration of the fistula 


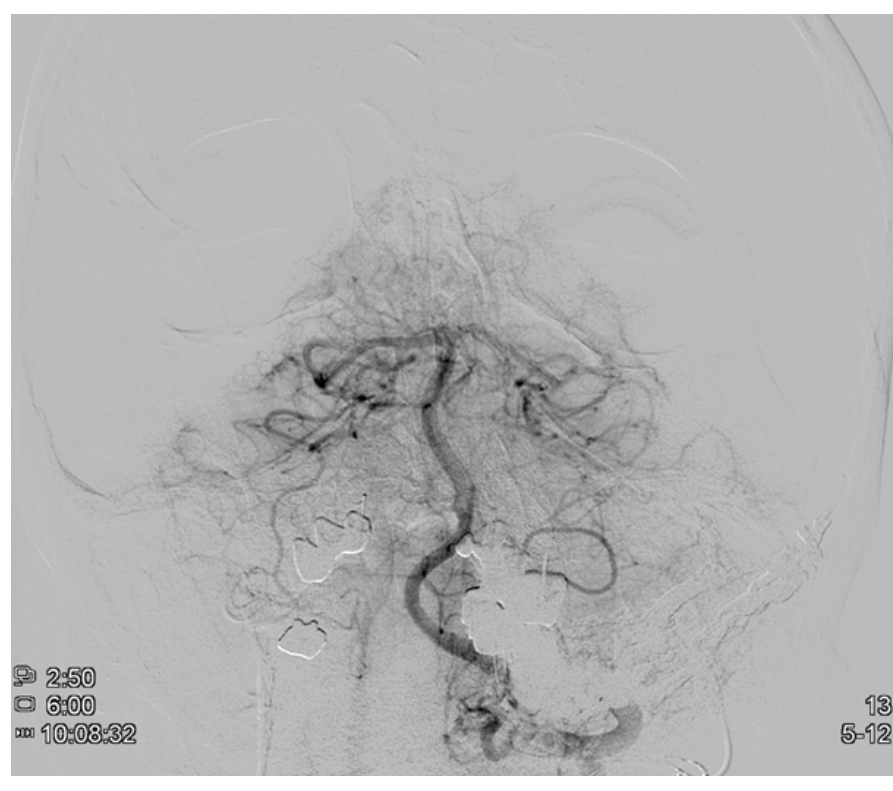

Figure 8. Anteroposterior LVA post-embolization DSA demonstrates obliteration of the fistula

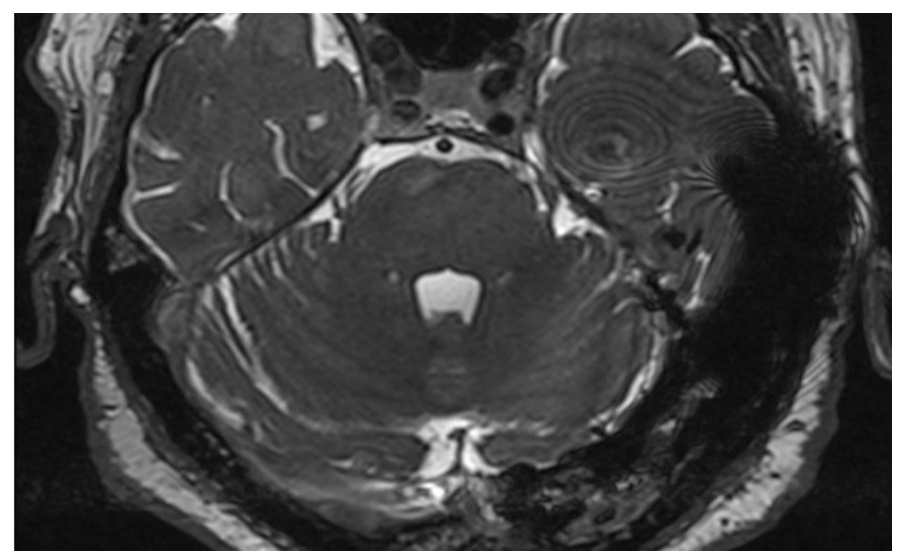

Figure 9. MRI (T2 weighted image) post embolization, at the follow up, shows the absence of abnormal veins at the root of left trigeminal nerve

\section{Discussion}

Trigeminal neuralgia (TN) is defined by recurrent unilateral brief electric shock-like pain that is abrupt in onset and termination. The pain is restricted to one or more of the trigeminal divisions and is triggered by innocuous sensory stimuli [5]. The compression of this cranial nerve at its root entry zone is the cause in most cases [6]. The most commonly identified vessels are the basilar artery, the SCA and the AICA [7]. Secondary, TN is usually caused by MS, tumors, aneurysms or very rare AVM. The dAVF presents an abnormal communication between arterial and venous vasculature at the dura matter of the brain and the spinal cord. It consists about $10-15 \%$ of all AVM and its presentation depends on the afferent and efferent vessels [8]. Because of the venous congestion and reflux, the dilated capillaries are vulnerable to hemorrhage either intraparechymatous or subarachnoid [9]. Also many other symptoms can be present due to venous compression or due to deep veins or sinuses thrombosis as headache, pulsatile tinnitus, ocular pain, ataxia and other brainstem deficits depending on its location and characteristics [10]. The dilated draining veins of the dAVF compress the trigeminal nerve at the root entry zone [11], causing symptoms and signs which is obvious in the pre-occlusion MRI scan of our patient.
Postoperatively, the improved radiological images are associated with the symptoms' improvement [12]. The treatment options of dAVF include endovascular embolization, surgical obliteration, stereotactic radiosurgery or a combination of the above [13]. The combined endovascular /surgical intervention has the highest probability of success for complete obliteration. The stereotactic radiosurgery requires months to years for occlusion to occur and is not indicated in patients with high risk features such as retrograde leptomeningeal venous drainage and venous congestion [14].

\section{Conclusion}

Trigeminal neuralgia is a severe, facial pain usually caused by vascular compression at the entry zone of the trigeminal nerve in the brainstem. When the pain persists, despite the intense and maximal medical treatment or other coexisting symptoms accompanies this prosopalgia, then further investigation for a rare, underlying cause is needed.

A dAVF, an AVM of the dura matter, is a very rare cause of the 5th nerve compression. It is presented with various symptoms and signs, reflecting venous congestion of brain and warning for fatal forthcoming consequences.

By suspecting a rare "curable" pathology we can offer a successful treatment, improving patient's quality of life, not only by relieving the unbearable pain but also by obviating the need for medication and avoiding potential side effects.

\section{Conflicts of Interest}

Sophia Vassilopoulou: no potential conflicts of interest with respect to the authorship and publication of this article.

Argyro Tountopoulou: no potential conflicts of interest with respect to the authorship and publication of this article.

Eleni Korompoki: no potential conflicts of interest with respect to the authorship and publication of this article. Loukia Poulou: no potential conflicts of interest with respect to the authorship and publication of this article.

Christos Gkogkas: no potential conflicts of interest with respect to the authorship and publication of this article.

\section{Contributions}

Sophia Vassilopoulou: drafting and critical revision of manuscript.

Argyro Tountopoulou: drafting and critical revision of the manuscript.

Eleni Korompoki: drafting and critical revision of the manuscript.

Loukia Poulou: drafting and critical revision of the manuscript.

Christos Gkogkas: drafting and critical revision of the manuscript.

\section{References}

1. Katusic S (1991) Epidemiology and clinical features of idiopathic trigeminal neuralgia and glossopharyngeal neuralgia: similarities and differences, Rochester, Minnesota, 1945-1984. Neuroepidemiology 10: 276-281.

2. Du R (2003) Trigeminal neuralgia in a patient with a dural arteriovenous fistula in Meckel's cave: case report. Neurosurgery, 53: 216-221.

3. Zakrzewska JM, Coakham HB (2012) Microvascular decompression for trigeminal neuralgia: update. Curr Opin Neurol 25: 296-301.

4. Ott D, Bien S, Krasznai L (1993) Embolization of a tentorial dural arterio-venous fistula presenting as atypical trigeminal neuralgia. Headache 33: 503-508. 
5. Maarbjerg S (2017) Trigeminal neuralgia - diagnosis and treatment. Cephalalgia 37: 648-657.

6. Mendelowitsch A, Radue EW, Gratzl O (1990) Aneurysm, arteriovenous malformation and arteriovenous fistula in posterior fossa compression syndrome. Eur Neurol 30: 338-342.

7. Maroon JC, Lunsford LD, Deeb ZL (1978) Hemifacial Spasm Due to Aneurysmal Compression of the Facial Nerve. Archives of Neurology 35: 545-546.

8. Lucas Cde P, Zabramski JM (2007) Dural arteriovenous fistula of the transversesigmoid sinus causing trigeminal neuralgia. Acta Neurochir 149: 1249-1253.

9. Cognard C (1995) Cerebral dural arteriovenous fistulas: clinical and angiographic correlation with a revised classification of venous drainage. Radiology 194: 671-680.
10. Chaichana KL (2012) Dural arteriovenous fistulas: epidemiology and clinical presentation. Neurosurg Clin N Am 23: 7-13.

11. Ge H (2016) Role of endovascular embolization for trigeminal neuralgia related to cerebral vascular malformation. Interv Neuroradiol 22: 600-605.

12. Rabinov JD (2013) ONYX versus n-BCA for embolization of cranial dural arteriovenous fistulas. J Neurointerv Surg 5: 306-310.

13. Lucas CP (1997) Treatment for intracranial dural arteriovenous malformations: a metaanalysis from the English language literature. Neurosurgery 40: 1119-1130.

14. Lu X (2014) Tentorial dural arteriovenous fistula manifesting as contralateral trigeminal neuralgia: resolution after transarterial Onyx embolization. $J$ Neurointerv Surg 6: 45.

Copyright: $(2020$ Vassilopoulou S. This is an open-access article distributed under the terms of the Creative Commons Attribution License, which permits unrestricted use, distribution, and reproduction in any medium, provided the original author and source are credited. 